Hautarzt 2016 $67: 4-5$

DOI 10.1007/s00105-015-3729-3

Online publiziert: 26. November 2015

(c) Springer-Verlag Berlin Heidelberg 2015

\title{
CrossMark
}

Die Fortschritte in der Grundlagenforschung und translationale Anstrengungen der letzten Jahrzehnte führten zur deutlichen Verbesserung der dermatologischen Diagnostik. Insbesondere die Erkenntnisse der molekularen Pathogenese haben zu einer rasanten Entwicklung von spezifischeren und empfindlicheren diagnostischen Tests erheblich beigetragen. Die Ära der molekularen Dermatologie hat dadurch seit einigen Jahren auch in den Praxisalltag Einzug gehalten. So finden die neuen Assays, die auf definierten Antigenen der pathogenen Erreger, Einzelallergene, Autoantigene bzw. Tumorantigene und Onkogene basieren, vermehrt Einsatz in der Routinediagnostik und bei der Definition von Ansatzpunkten von modernen Therapien von Infektionen, Allergien, Autoimmundermatosen und Hauttumoren. Im aktuellen Leitthemenheft werden Updates zur dermatologischen Labordiagnostik in verschiedenen Bereichen vorgestellt.

$>$ Wir sind in der Ära der molekularen dermatologischen Diagnostik angekommen.

Es ist aus zahlreichen Studien der letzten Jahre bekannt, dass die Inzidenz und Prävalenz der Geschlechtskrankheiten, Allergien, Autoimmundermatosen und des Melanoms deutlich zugenommen haben. Auch werden durch molekulare Marker neue Krankheitsentitäten definiert und erkannt. Die neue molekulare Diagnostik in diesen Bereichen ist entscheidend für die Frühdiagnose und Risikoeinschätzung sowie für Verlaufsund Therapiemonitoring und ermöglicht somit ein effizientes Management unserer

C. Sitaru ${ }^{1,2} \cdot$ J. Bauer ${ }^{3}$

${ }^{1}$ Klinik für Dermatologie und Venerologie, Universitätsklinikum Freiburg, Freiburg, Deutschland

${ }^{2}$ MVZ Labor Clotten, Freiburg, Deutschland

${ }^{3}$ Universitäts-Hautklinik Tübingen, Tübingen, Deutschland

\section{Moderne Labordiagnostik in der Dermatologie}

Patienten in einer Zeit immer knapper werdender Ressourcen im Gesundheitswesen.

In dem Artikel von Brockmeyer und Meyer werden die aktuellen Ansätze in der Diagnostik von Geschlechtskrankheiten (STI) vorgestellt. Der Einsatz von Nukleinsäureamplifikationstests, die sog. NAATs, ist nicht nur für die Detektion der STI-Erreger wichtig, sondern kann auch als genotypische Resistenztestung eingesetzt werden. Darüber hinaus erlauben neue Sequenziertechniken den raschen Nachweis von resistenten Erregern, auch wenn diese in einer heterogenen Population vergleichsweise selten sind.

Als Dermatologen sind wir im Alltag mit verschiedenen klinischen Manifestationsformen mukokutaner Mykosen konfrontiert, die eine schnelle und zuverlässige Diagnostik erfordern, bevor eine angemessene Therapie eingeleitet wird. Die Verzahnung von Klinik, Diagnostik und Therapie ermöglicht eine optimale Interpretation der klinischen und Laborbefunde. Die mykologische Diagnostik erfordert hohe Standards des Qualitätsmanagements, die nach dem Inkrafttreten der neuen Richtlinien zur Qualitätssicherung laboratoriumsmedizinischer Untersuchungen durch die Bundesärztekammer vielerorts unter Dermatologen zur Unsicherheit geführt haben, wie diese qualitätssichernden Maßnahmen im Bereich der Mykologie umgesetzt werden können. Der Beitrag von Schaller et al. adressiert diese Aspekte der Qualitätssicherung für die Kollegen, die mykologische Labordiagnostik in ihrer Praxis durchführen.

Die zunehmende Prävalenz von Allergien stellt eine weitere Heraus- forderung bei der Diagnose und dem Management der Patienten dar. Die Invitro-Diagnostik ist neben der Anamnese, den Prick-, Intrakutan- und Epikutantestungen eine wichtige Säule der Allergiediagnostik. Die Bestimmung von spezifischen IgE ermöglicht durch die Verwendung von molekularen Allergenkomponenten und Allergen-Arrays eine präzise Klassifikation für die Einschätzung der Schwere und das Risikomanagement von Allergien. Auch zelluläre Tests wie der Basophilenaktivierungstest oder der Lymphozytentransformationstest können die Sensitivität der Diagnostik erhöhen.

Der Nachweis von gewebsgebundenen und zirkulierenden Autoantikörpern ist von zentraler Bedeutung für die Diagnostik der blasenbildenden Dermatosen, Kollagenosen oder Vaskulitiden. Ein profundes Verständnis der molekularen Spezifität der Autoantikörper und deren klinischen Assoziation ist für die exakte Diagnose unentbehrlich. Wichtig in der täglichen Praxis ist auch eine schnelle Orientierung hinsichtlich der Autoantikörper bzw. Erkrankungsmarker, die sich für die quantitative Bestimmung als Verlaufsparameter eignen. Diese und andere Aspekte der modernen, leitliniengerechten Diagnostik von Autoimmundermatosen werden in den entsprechenden Beiträgen von Hoffmann et al. vorgestellt.

Die moderne molekulare Diagnostik des Melanoms wird im Artikel von Bauer dargestellt. Dank der Aufklärung molekularer Mechanismen der Melanomentstehung kam es in den letzten Jahren zu einem Paradigmenwechsel. Noch vor wenigen Jahren war das fortgeschrittene Melanom kaum für Therapien zugänglich. Die Ent- 
deckung von wichtigen Signalwegen und Mutationen im Melanom und die Entwicklung von zielgerichteten Therapien führen heute zu einem guten Ansprechen vieler Patienten. Die Mutationsanalyse von fortgeschrittenen Melanomen stellt damit Grundlage für die individualisierte Therapie mit mutationsspezifischen Inhibitoren z. B. gegen mutiertes BRAF dar. Auch bei der Diagnose prognostisch unklarer melanozytärer Tumoren kann die Analyse von Veränderungen im Tumorgenom mittels komparativer genomischer Hybridisierung (CGH) oder Fluoreszenzin-situ-Hybridisierung (FISH) wertvolle Informationen über die genomische Stabilität des Tumors liefern.

Auch im Bereich der Genodermatosen, die selten vorkommen und sich mit einem breiten Spektrum an kutanen und extrakutanen Symptomen manifestieren, ist neben der klinischen Bewertung auch eine molekularbiologische Diagnostik unabdingbar. Durch klar definierte diagnostische Pfade sowie entsprechende Kooperation mit der Humangenetik sollen unbegründete und unnötig umfangreiche genetische Analysen vermieden und eindeutige Diagnosen gestellt werden.

Durch die kontinuierliche Weiterentwicklung unserer klinischen und labordiagnostischen Kompetenzen können wir die Versorgung unserer Patienten im vielfältigen Fach Dermatologie verbessern. Wir wünschen Ihnen daher eine interessante Lektüre und hoffen, dass Sie auch im Alltag von den neuen, molekularen Methoden profitieren werden.

Prof. Dr. J. Bauer

Prof. Dr. C. Sitaru

\section{Korrespondenzadresse}

Prof. Dr. C. Sitaru

MVZ Labor Clotten

Merzhauserstr. 112A, 79100 Freiburg

cassian@mail.sitaru.eu

\section{Prof. Dr. J. Bauer}

Universitäts-Hautklinik Tübingen Liebermeisterstraße 25, 72076 Tübingen

juergen.bauer@med.uni-tuebingen.de

Interessenkonflikt. C. Sitaru und J. Bauer geben an, dass kein Interessenkonflikt besteht.
Jörg Kleine-Tebbe und Thilo Jakob (Hrsg.)

\section{Molekulare Allergiediagnostik}

Springer 2015, 392 S., (ISBN 978-3-66245220-2), 49,99 EUR

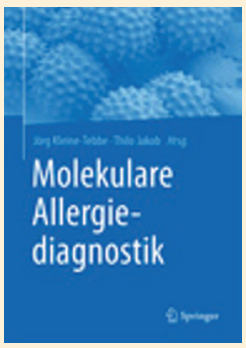

Molekulare Allergiediagnostik im klinischen Alltag. Leben heißt lernen, lebenslang lernen. Die rasante Entwicklung der molekularen Allergologie in den letzten

Jahren führt uns Ärzten diesen Aphorismus einmal wieder überdeutlich vor Augen. Dabei lernen wir nicht nur für uns selbst, sondern primär für unsere Patienten, damit diese im klinischen Alltag von optimierter Diagnostik und Therapie profitieren können.

Viele Allergologen sind mit der Messung der serologischen lgE-Reaktivität gegen Allergenextrakte groß geworden. In dem Wissen, dass sowohl Sensitivität als auch Spezifität naturgemäß begrenzt sein müssen, denn Allergenextrakte enthalten neben den eigentlichen Allergenen unzählige nicht-allergene Substanzen. In den vergangenen Jahrzehnten wurden mehr als tausend verschiedene Einzelallergene molekular charakterisiert. Diese definierten allergenen Moleküle werden für diagnostische Zwecke entweder aus natürlichem Allergenmaterial isoliert oder werden rekombinant hergestellt. Um die verbesserte Sensitivität der molekularen Diagnostik im klinischen Alltag gewinnbringend einzusetzen, sind umfangreiche und detaillierte Kenntnisse der molekularen Allergologie erforderlich.

Ein entsprechender Leitfaden, um sich im Labyrinth der vielen Einzelallergene zurechtzufinden, fehlte bisher. Diese Lücke wurde dann als besonders schmerzhaft empfunden, wenn man sich seine Informationen zur molekularen Allergologie aus verschiedensten Publikationen und Monographien mühsam zusammensuchen musste. Das Buch Molekulare Allergiediagnostik füllt jetzt nicht nur diese Lücke, sondern erfüllt seinen Zweck als Leitfaden durch das "Einzelallergen-Labyrinth" ganz hervorragend. Die Molekulare Allergiediagnostik beginnt mit einer Einführung und Systematik der wichtigsten Proteinfamilien. Der Abschnitt zu den Testsystemen hilft dem Leser bei der
Interpretation der Befunde und der Ermittlung der klinischen Relevanz. Auch der nächste Abschnitt zur molekularen Allergiediagnostik im klinischen Alltag ist rundum gelungen. Hier wird der Stellenwert der molekularen Diagnostik detailliert dargestellt, sowohl bezüglich einzelner Allergengruppen und verschiedenster Nahrungsmittel als auch hinsichtlich bestimmter Krankheitsbilder. Die Autoren zeigen, wie Kenntnisse zu Einzelallergenen in das bisherige Konzept einer speziesbezogenen Allergenkunde integriert werden, was unter anderem die Differenzierung von multiplen Sensibilisierungen und Kreuzallergien erlaubt. Jedes der Kapitel endet mit einem Fazit für den klinischen Alltag, das alle relevanten Informationen noch einmal lesefreundlich und didaktisch wertvoll aufbereitet. Es zieht sich wie ein roter Faden durch das ganze Buch, dass die Autoren nicht nur alle bekannten Fakten und Daten darlegen möchten, sondern gleichzeitig ganz besonders viel Mühe darauf verwenden, dem Leser die klinische und praktische Relevanz der neuen Erkenntnisse nahezubringen.

Das Buch Molekulare Allergiediagnostik möchte ich daher allen Allergologen, auch den „alten Hasen", uneingeschränkt empfehlen, auch von allen Ärzten/Innen in Weiterbildung sollte es rege benützt werden. Ich kann Ihnen versprechen, dass Sie durch Lesen und Stöbern in dem Buch Molekulare Allergiediagnostik viel Wissen für Ihren praktisch-klinischen Alltag erwerben!

Axel Trautmann (Würzburg) 